\title{
ASYMPTOTIC VALUE-AT-RISK ESTIMATES FOR SUMS OF DEPENDENT RANDOM VARIABLES
}

BY

\author{
MARIO V. WÜTHRICH
}

\begin{abstract}
We estimate Value-at-Risk for sums of dependent random variables. We model multivariate dependent random variables using archimedean copulas. This structure allows one to calculate the asymptotic behaviour of extremal events. An important application of such results are Value-at-Risk estimates for sums of dependent random variables.
\end{abstract}

\section{KEYWORDS}

Archimedean Copula, Dependent Risks, Value-at-Risk, Extreme Value Theory, Pickands-Balkema-de Haan Theorem, Multivariate Extremes.

\section{INTRODUCTION AND Motivation}

Consider a portfolio consisting of $d$ similar insurance policies. The aggregate claim $S$ of the portfolio is the sum of all single claims $X_{1}, \ldots, X_{d}$, i.e.

$$
S=\sum_{i=1}^{d} X_{i}
$$

The calculation of the premium and a risk management analysis are two of the main topics of risk theory. Therefore not only the marginals of the $X_{i}$ 's have to be known but also their dependence structure. In practice, this problem is often simplified by assuming that the random variables $X_{i}$ are independent and identical distributed (i.i.d.). Of course, the independence hypothesis is a very strong assumption which is almost never satisfied. In the present paper we model a portfolio consisting of $d$ dependent risks. We use the copula framework to model the dependence structure and we will see that such a dependence structure may have an enormous effect on Value-at-Risk considerations.

Copulas were originally introduced in 1959 in the context of probabilistic metric spaces (see Sklar [16] and Schweizer-Sklar [15]). During the past years they have developped rapidly and they have attracted much interest. Copulas are used to describe scale invariant dependencies between random variables. An understanding of such stochastic dependence structures has become very 
important in many fields of probability theory. Copulas have been particularly useful for constructing appropriate mulitivariate models in the areas of modern risk management and stress testing in the actuarial world and in finance. An overview of recent developments and applications can be found in Joe [7], Nelsen [14], Frees-Valdez [5], Juri-Wüthrich [8, 9], Embrechts-McNeil-Straumann [4] and the references therein.

Nevertheless it is quite difficult to fit copulas to real data (for successful real data fitting of copulas by actuaries see Klugman-Prasa [11] and Hürlimann [6]). It is difficult to have a good intuitive feeling for dependence structures (see e.g. fallacies in [4] and [9]). Moreover, in extreme value theory, one does not usually have enough data to analyze the behaviour of extremal events. Therefore it is very important to have appropriate convergence theorems, in order to approximate tail probabilities by their asymptotic values. This procedure is applied quite often in practice: if we know the asymptotic behaviour we do not need to worry about the exact choice of the model, but only about the estimation of parameters and the question of speed of convergence.

In the present article we restrict ourselves to exchangeable random variables with archimedian copulas. Among this class of random variables we discover a certain "universality" which makes it easy to derive asymptotic Value-at-Risk estimates for aggregate random variables. These asymptotic estimates follow from the asymptotic copula behaviour which is measured in terms of regular variation. We prove weak convergence results (see Lemmas 6.1, 7.1, 7.2 below) from which we can derive asymptotic Value-at-Risk estimates (see Theorem 3.3 below).

The use of these results for risk managers and actuaries is similar to that of e.g. the central limit theorem for sums of random variables or the Fisher-Tippett theorem for extremal events (see [3], Theorem 3.2.3): having only partial information on the model we are able to give estimates for the Value-at-Risk with the help of our asymptotic results. The questions we do not study here are 1) Speed of convergence and approximation error terms; 2) Validation and justification of our model assumptions (Assumption 3.1). One should carefully consider the exchangeability (indistinguishability) assumption: we give some arguments justifying the sense of our model. 3) Similar results (to Theorem 3.3) for non-archimedean copulas.

Organization of this paper: In the next section we introduce the concept of copulas. These copulas describe multivariate dependence structures of random variables which are studied in the sequel. In Section 3 we present our main result, Theorem 3.3, which describes asymptotic quantile behaviours. In Section 4 we identify the limiting constants and in Section 5 we give examples. Finally in the remaining part we prove all our results. The crucial steps are the weak convergence statements given in Lemmas 6.1, 7.1, 7.2.

\section{Multivariate Copulas}

The idea behind the concept of copulas is to separate a multivariate distribution function into two parts, one describing the dependence structure and the other one describing marginal behaviours, respectively. 
Definition 2.1 (Copula) Choose $d \geq 2$. A d-dimensional copula is a d-dimensional distribution function restricted to $[0,1]^{d}$ with uniform- $(0,1)$ marginals.

Theorem 2.2 (Sklar [16], [15], [14]) For a given joint distribution function $F$ with continuous marginals $F_{1}, \ldots, F_{d}$ there exists a unique copula $C$ satisfying

$$
F\left(x_{1}, \ldots, x_{d}\right)=C\left(F_{1}\left(x_{1}\right), \ldots, F_{d}\left(x_{d}\right)\right) .
$$

Conversely, for a given copula $C$ and marginals $F_{1}, \ldots, F_{d}$ we have that (2.1) defines a distribution with marginals $F_{i}$.

Sklar's theorem is a motivation for calling a copula a dependence structure. In fact, (2.1) means that $C$ couples the marginals $F_{i}$ to the joint distribution function $F$. One important property of copulas is that the copula of a random vector $\left(X_{1}, \ldots, X_{d}\right)$ is invariant under strictly increasing transformations.

There are several special copulas, e.g. the so called comonotonic copula $C_{U}\left(x_{1}, \ldots, x_{d}\right)=\min \left\{x_{1}, \ldots, x_{d}\right\}$ which corresponds to total positive dependence or the independent copula which is the copula of independent random variables: $\mathrm{C}_{\mathrm{I}}\left(x_{1}, \ldots, x_{d}\right)=x_{1} \cdot \ldots \cdot x_{d}$ (for more background information we refer to $[4,7,14]$ ). In this article we focus on archimedean copulas.

Definition 2.3 Choose $d \geq 2$. Let $\psi:[0,1] \rightarrow[0, \infty]$ be strictly decreasing, convex and such that $\psi(0)=\infty$ and $\psi(1)=0$. Define for $x_{i} \in[0,1], i=1, \ldots, d$

$$
C^{\psi}\left(x_{1}, \ldots, x_{d}\right)=\psi^{-1}\left(\sum_{i=1}^{d} \psi\left(x_{i}\right)\right) .
$$

The function $\psi$ is called generator of $C^{\psi}$.

Remark. For $d \geq 3, C^{\psi}$ is in general not a copula. There are generators such that $C^{\psi}$ is not a distribution (see Kimberling [10] or Nelsen [14], Section 4.6). Henceforth we give necessary and sufficient conditions for $\psi$ under which $C^{\psi}$ is a copula for all $d \geq 2$ (see Nelsen [14], Section 4.6). We say: $\psi^{-1}$ is completely monotonic on $[0, \infty)$ if for all $k \in \mathbb{N}, x>0$,

$$
(-1)^{k} \frac{d^{k}}{d x^{k}} \psi^{-1}(x) \geq 0 .
$$

The following theorem gives necessary and sufficient conditions for having a copula (see Nelsen [14], Theorem 4.6.2).

Theorem 2.4 (Kimberling [10]) $C^{\psi}$ is a copula for all $d \geq 2$ if and only if the generator $\psi$ has an inverse $\psi^{-1}$ which is completely monotonic on $[0, \infty)$.

Remark. If $\psi^{-1}$ is completely monotonic on $[0, \infty)$ we call $C^{\psi}$ archimedean copula. Archimedean copulas are interesting in practice because they are very easy to construct, but still we obtain a rich family of dependence structures. Usually 
they have only one parameter which is a great advantage when one needs to estimate parameters from data.

\section{Examples of archimedean copulas:}

- The Clayton copula with $\alpha>0$ is generated by $\psi(t)=t^{-\alpha}-1$ and takes the form

$$
C^{\mathrm{Cl}, \alpha}\left(x_{1}, \ldots, x_{d}\right)=\left(x_{1}^{-\alpha}+\ldots x_{d}^{-\alpha}-d+1\right)^{-1 / a} .
$$

- The generator $\psi(t)=e^{\theta / t}-e^{\theta}$ with $\theta>0$ corresponds to

$$
C^{\psi, \theta}\left(x_{1}, \ldots, x_{d}\right)=\theta / \log \left\{\sum_{i=1}^{d} e^{\theta / x_{i}}-(d-1) e^{\theta}\right\} .
$$

Further examples may be constructed as follows. For a generator $\psi$ and $\kappa \geq 1$

$$
\phi(t)=\psi^{k}(t)
$$

is a generator too.

\section{Quantiles for Sums of Dependent Random Variables}

In this section we give our main results and a classification of the multivariate distributions. In view of Sklar's theorem we are able to decouple the dependence structure from the marginals, therefore we can analyze their behaviours separately.

In Subsection 3.1 we define our model and we give a definition of regular variation which is used for the characterization of the asymptotic behaviour of extremal events.

In Subsection 3.2 we give a classification of the marginals. It should be pointed out that all "sufficiently nice" distributions belong to one of these three classes from an extreme value point of view. I.e. extremal events can be characterized by three different types of behaviour (limit laws).

Finally in Subsection 3.3 we characterize the dependence structures and give our main results.

\subsection{Definitions and assumptions}

Let us first give the crucial assumption for our results:

Assumption 3.1 Assume that the random vector $\mathbf{X}=\left(X_{1}, \ldots, X_{d}\right), d \geq 2$, satisfies:

1. Every coordinate $X_{i}$ has the same continuous marginal $F(x)=P\left[X_{i} \leq x\right]$.

2. $\mathbf{X}=\left(X_{1}, \ldots, X_{d}\right)$ has archimedean copula $C^{\psi}$. 


\section{Remarks:}

- Assumption 3.1 is quite natural if we think that we have a portfolio of exchangeable (indistinguishable) dependent risks. One should also mention that due to fact that copulas are invariant under strictly increasing transformations (see remark after Theorem 2.2) it is always possible to transform the random vector such that it has identical marginal distributions.

- In practice, we are often in the situation where the random variables are not exchangeable and where we do not want to transform them. In our context (from an extreme value or risk management point of view) non-exchangeable situations are only of interest if the marginals have the same asymptotic behaviours (see Subsection 3.2). Otherwise one finds that one of the coordinates is dominating the other ones (see Lemmas 6.1, 7.1, 7.2 below).

- Here, we restrict ourselves to archimedean copulas. One could generalize our results to other copulas. We have done this generalization in a different context (see [8] and [9]) and we have seen that the notations and calculations become much more complicated in the general situation. To keep the analysis of this article easy we have decided to restrict ourselves to archimedean copulas. By the way, also among archimedean copulas there are interesting ones from an extreme value point of view. Especially, we have seen (see [8], Section 3.2) that the Clayton copula plays a crucial role in multivariate extreme value theory.

- There is a wide range of examples where one has a portfolio of similar dependent risks and where one wants to study tail probabilities of the form (1.1): e.g. travel insurance or health insurance portfolio (see e.g. BäuerleMüller [1]), credit risk portfolio (see e.g. Frey-McNeil [13]), reinsurance portfolio (see e.g. Kremer [12]), Motor hull portfolio with protection against hail storms, etc.

We will be interested into the limit $x \downarrow x_{F}$, where $x_{F}=\inf \{x \in \mathbb{R} ; F(x)>0\}$ denotes the left endpoint of the distribution $F$. We have seen (see e.g. [9]) that from a mathematical point of view it is much easier to analyze lower tail dependence than upper tail dependence. Therefore we assume that losses $X_{i}$ are negative and that we are interested into the behaviour $X_{i} \rightarrow-\infty, x_{F}$ resp. In practice, this can always be obtained by 'sign flips' $(x \mapsto-x)$. Such a 'sign flip' reflects a transformation from a random vector $\mathbf{X}$ with copula $C$ to a random vector $-\mathbf{X}$ with copula $\hat{C}$, where $\widehat{C}$ is the survival copula of $C$ (see [9]). Such a transformation reflects also changing from upper tail dependence to lower tail dependence.

Definition 3.2 A function $f$ is regularly varying with index $\alpha \in \mathbb{R}$ at $x^{-}\left(x^{+}\right.$, resp.) if for all $t>0$

$$
\lim _{y \uparrow x} \frac{f(y t)}{f(y)}=t^{\alpha}, \quad \lim _{y \downarrow x} \frac{f(y t)}{f(y)}=t^{\alpha}, \text { resp. }
$$

We write $f \in \mathfrak{R}_{\alpha}^{x^{-}}\left(f \in \mathfrak{R}_{\alpha}^{x^{+}}\right.$, resp.). For $\alpha=0$ we say $f$ is slowly varying. For $\alpha=-\infty$ (i.e. we consider for the rhs of (3.1) $\lim _{\alpha \rightarrow-\infty} t^{\alpha}$ ) we use the terminology 
rapidly varying. A standard reference on regular variation is Bingham-GoldieTeugels [2].

\subsection{Marginal behaviours}

As already mentioned above there are three different types of marginal behaviours: In the Fisher-Tippett Theorem and the Pickands-Balkema-de Haan Theorem (see e.g. Theorems 3.2.3 and 3.4.13(b) in [3]) one sees that the one-dimensional distributions (marginals) can essentially be divided into three subclasses for analyzing extremal events. The crucial conditition is the rate of decay at $\pm \infty$, i.e. the fatness of the tails: Let $x_{F}$ denote the left-endpoint of the distribution $F$. Then the three subclasses are characterized by (see Theorems 3.3.7, 3.3.12 and 3.3.27 in [3] for necessary and sufficient conditions)

- Fréchet case: $F(\cdot) \in \mathfrak{R}_{-\beta}^{-\infty}$ for $\beta>0$.

- Weibull case: Assume $x_{F}>-\infty$ and $F\left(x_{F}-1 / \cdot\right) \in \mathfrak{R}_{-\beta}^{-\infty}$ for $\beta>0$.

- Gumbel case: Assume $x_{F}>-\infty$ and there exists a positive function $a(\cdot)$ such that for $t \in \mathbb{R}$

$$
\lim _{u \downarrow x_{F}} \frac{F(u+t a(u))}{F(u)}=e^{t} .
$$

For a possible choice of $a(\cdot)$ we refer to [3], (3.34).

We want to stress here once more that in applied problems one can often not give an explicit description of the excess distribution for rare events (since one has almost no datas). Therefore it is often a successful way to approximate the true distribution by one of the three limit laws (Fréchet, Weibull or Gumbel distribution) since we know that asymptotically we have a weak convergence result.

Examples are given below, see Section 5.1.

\subsection{Main theorem}

As for the marginals, there are exactly three different types of behaviours for archimedean copulas (see also [8], Section 3.1). We can divide archimedean copulas into the following three subclasses: a) regularly varying ones, b) rapidly varying ones (comonotonic copula), and c) slowly varying ones. In this article we only treat subclass a) (regularly varying ones). The claims and proofs for b) (rapidly varying ones) are similar to the regularly varying case. Case c), the slowly varying case, is more complicated since it ranges from independent to weakly tail dependent copulas (see also Fallacy 5.1 in [9]). Especially, it is necessary to analyze higher order terms in order to determine asymptotic behaviours. We will not treat this case here because it would go beyond the scope of this article. 
Theorem 3.3 Assume $d \geq 2, \alpha>0$ and $\beta>0$. There exist constants $q_{d}^{F}(\alpha, \beta), q_{d}^{W}(\alpha$, $\beta), q_{d}^{G}(\alpha) \in\left[d^{-1 / \alpha}, \infty\right)$ such that for all $\mathbf{X}$ satisfying Assumption 3.1 with $\psi \in \mathfrak{R}_{-\alpha}^{0+}$ and

a) Fréchet case: with marginals $F(\cdot) \in \mathfrak{R}_{-\beta}^{-\infty}$ :

$$
\lim _{u \rightarrow \infty} \frac{1}{F(-u)} P\left[\sum_{i=1}^{d} X_{i} \leq-u d\right]=q_{d}^{F}(\alpha, \beta) .
$$

b) Weibull case: with marginals $F$ with $x_{F}>-\infty$ and $F\left(x_{F}-1 / \cdot\right) \in \mathfrak{R}_{-\beta}^{-\infty}$.

$$
\lim _{u \rightarrow \infty} \frac{1}{F\left(x_{F}+1 / u\right)} P\left[\sum_{i=1}^{d} X_{i} \leq d x_{F}+d / u\right]=q_{d}^{W}(\alpha, \beta) .
$$

c) Gumbel case: with marginals $F$ with $x_{F} \geq-\infty$ and there exists a positive function $a(\cdot)$ such that (3.2) is satisfied, we have

$$
\lim _{u \downarrow x_{F}} \frac{1}{F(u+a(u))} P\left[\sum_{i=1}^{d} X_{i} \leq d(u+a(u))\right]=q_{d}^{G}(\alpha) .
$$

\section{Interpretation and Properties.}

- The constants in the above theorem can explicitly be calculated. The explicit forms of the limiting constants are given below in formula (4.6).

- Theorem 3.3 shows that $\alpha$ and $\beta$ determine the asymptotic behaviour of aggregate random variables (e.g. in the Fréchet case): Out of $\alpha$ and $\beta$ we calculate the constant $q_{d}^{F}(\alpha, \beta)$. Hence $d, \alpha$ and the marginal $F$ completely determine the asymptotic behaviour of our aggregate random variables. For practical purposes this means that we do not need to worry about an explicite choice of the archimedean dependence structure. As long as we can determine the strength of dependence $\alpha$ we can exactly determine the asymptotic behaviour. For very small quantiles (e.g. Value-at-Risk estimates) we approximate then the 'true' quantile by:

$$
P\left[\sum_{i=1}^{d} X_{i} \leq-u d\right] \sim F(-u) \cdot q_{d}^{F}(\alpha, \beta), \quad \text { as } u \rightarrow \infty .
$$

\section{A ReCursive Formula for $q_{d}(\alpha, \beta)$}

In this section we define two families of distributions which turn out to be useful for two purposes. As we will see in Section 6 and 7 they will be used to prove our main theorem. On the other hand their quantiles allow for an explicit calculation of the constants $q_{d}^{L}(\alpha, \beta), L=F, W, G$.

Choose $\alpha, \beta>0, k, d \in \mathbb{N}$ with $1 \leq k \leq d$ and $d \geq 2$. Then we define for $x_{1}, \ldots, x_{k}$ $\in[0,1]$ and $x_{k+1}, \ldots, x_{d} \geq 0$ 


$$
H_{d, k}^{(\alpha, \beta)}\left(x_{1}, \ldots, x_{d}\right)=\left(\frac{\sum_{i=1}^{d} x_{i}^{-\alpha \beta}}{k}\right)^{-1 / \alpha}
$$

For $x_{1}, \ldots, x_{k} \leq 1$ and $x_{k+1}, \ldots, x_{d} \in \mathbb{R}$ we define

$$
G_{d, k}^{(\alpha)}\left(x_{1}, \ldots, x_{d}\right)=\left(\frac{\sum_{i=1}^{d} \exp \left\{-\alpha\left(x_{i}-1\right)\right\}}{k}\right)^{-1 / \alpha} .
$$

Lemma 4.1 $H_{d, k}^{(\alpha, \beta)}$ defines a continuous distribution on $[0,1]^{k} \times[0, \infty)^{d-k}$. The function $G_{d, k}^{(\alpha)}$ defines a continuous distribution on $(-\infty, 1]^{k} \times \mathbb{R}^{d-k}$. Both distributions have Clayton copula.

Remark: In view of [8] it is not surprising that both distributions have Clayton copula. This comes from the fact that the Clayton copula is invariant under certain transformations and plays the role of an attracting copula (see [9]).

Now we are able to define our constants. We choose two random vectors $\left(Z_{1}^{(k)}, \ldots, Z_{d}^{(k)}\right) \stackrel{d}{\sim} H_{d, k}^{(\alpha, \beta)}$ and $\left(\tilde{Z}_{1}^{(k)}, \ldots, \tilde{Z}_{d}^{(k)}\right) \stackrel{d}{\sim} G_{d, k}^{(\alpha)}$. Then we define the constants

$$
\begin{aligned}
& q_{d, k}^{F}(\alpha, \beta)=P\left[\sum_{i=1}^{d} 1 / Z_{i}^{(k)} \geq d\right]>0 \\
& q_{d, k}^{W}(\alpha, \beta)=P\left[\sum_{i=1}^{d} Z_{i}^{(k)} \leq d\right]>0 \\
& q_{d, k}^{G}(\alpha)=P\left[\sum_{i=1}^{d} \tilde{Z}_{i}^{(k)} \leq d\right]>0 .
\end{aligned}
$$

The constants in Theorem 3.3 are then given by $(L=F, W, G)$

$$
q_{d}^{L}(\alpha, \beta)=\sum_{k=1}^{d}(-1)^{k-1}\left(\begin{array}{l}
d \\
k
\end{array}\right) q_{d, k}^{L}(\alpha, \beta) \cdot k^{-1 / \alpha}<\infty .
$$

Remark: For some parameters $\alpha, \beta$ and $d$, we can explicitly calculate these constants (see Subsection 5.2), for all remaining cases we can at least numerically determine the constants $q_{d}^{L}(\alpha, \beta)$.

An alternative descriptions for $q_{d}^{L}(\alpha, \beta)$ can be found via the following identity: Assume $\left(Z_{1}^{(1)}, \ldots, Z_{d}^{(1)}\right) \stackrel{d}{\sim} H_{d, 1}^{(\alpha, \beta)}$ then for $x_{i} \leq 1(i=1, \ldots k)$

$$
\begin{aligned}
& P\left[Z_{1}^{(1)} \leq x_{1}, \ldots, Z_{d}^{(1)} \leq x_{d} \mid Z_{1}^{(1)} \leq 1, \ldots, Z_{k}^{(1)} \leq 1\right] \\
& =\frac{H_{d, 1}^{(\alpha, \beta)}\left(x_{1}, \ldots, x_{d}\right)}{H_{d, 1}^{(\alpha, \beta)}(1, \ldots, 1, \infty, \ldots, \infty)}=H_{d, k}^{(\alpha, \beta)}\left(x_{1}, \ldots, x_{d}\right) .
\end{aligned}
$$

From this one also easily sees that $q_{d, d}^{L}(\alpha, \beta)=1$. 


\section{EXAMPLES AND CONCLUSIONS}

\subsection{Marginal behaviours}

In [3], p. 153, there is an overview of the most common marginal distributions in each class. We choose one explicit example in the Fréchet and in the Gumbel case.

- The Fréchet case contains heavy-tailed distributions like the Pareto, Cauchy, Burr or loggamma distribution. Here we concentrate on the Pareto distribution: For $\theta, \beta>0$ and $x \leq-\theta$ we have

$$
F(x)=(\theta /-x)^{\beta} \in \Re_{-\beta}^{-\infty} .
$$

- The Weibull case contains short-tailed distributions with finite support like the uniform or beta distribution.

- The Gumbel case contains moderately heavy to light-tailed distributions like the exponential, gamma, normal or lognormal distribution. As an example we choose the standard Gaussian distribution: Using Mill's ratio (see e.g. [3], Example 3.3.29), we obtain

$$
\Phi(x) \sim \frac{1}{(2 \pi)^{1 / 2}} \cdot \frac{1}{|x|} \cdot e^{-x^{2} / 2} \quad \text { as } x \rightarrow-\infty .
$$

\subsection{Value-at-Risk estimates}

In this subsection we choose explicit examples for which we calculate the asymptotic Value-at-Risk. If losses are negative, the Value-at-Risk at level $1-p$ of a random variable $X$ is defined for $p \in(0,1)$ as

$$
\operatorname{VaR}_{1-p}(X)=-\sup \{x \in \mathbb{R} ; P[X \geq x] \geq 1-p\} .
$$

Typically we are interested into $p$ small. Formula (5.3) is just the "usual" definition of $\operatorname{VaR}_{1-p}(-X)$.

In Theorem 3.3 we have seen that one does not have to make an explicit choice for the generator $\psi$. All we have to choose is $\alpha>0$. One explicit choice would be e.g. the Clayton copula $\psi(t)=t^{-\alpha}-1 \in \mathfrak{R}_{-\alpha}^{0+}$. For more examples of copulas with regularly varying generator we also refer to [8].

In order to determine the asymptotic behaviour of the quantiles we need to evaluate the constants in Theorem 3.3. To make our lives easy we choose $d=2$. Hence for $L=F, W, G$ we have (see (4.6))

$$
q_{2}^{L}(\alpha, \beta)=2 \cdot q_{2,1}^{L}(\alpha, \beta)-2^{-1 / \alpha} .
$$

Hence we need to calculate $q_{2,1}^{L}(\alpha, \beta)$. 


\section{Example 5.1 (Fréchet case)}

Consider $-X_{1}$ and $-X_{2}$ with Pareto marginals with parameter $\beta \geq 1$ and archimedean copula generated by $\psi \in \mathfrak{R}_{-\alpha}^{0+}$ with $\alpha>0$. In view of (4.3):

$$
\begin{aligned}
q_{2,1}^{F}(\alpha, \beta) & =P\left[\sum_{i=1}^{2} \frac{1}{Z_{i}^{(1)}} \geq 2\right]=P\left[Z_{1}^{(1)} \leq 1 / 2\right]+P\left[Z_{1}^{(1)}>1 / 2, Z_{2}^{(1)} \leq \frac{Z_{1}^{(1)}}{2 Z_{1}^{(1)}-1}\right] \\
& =(1 / 2)^{\beta}+\left.\int_{1 / 2}^{1} \frac{d H_{2,1}^{(\alpha, \beta)}(x, y)}{d x}\right|_{(x, y)=\left(x_{1}, 2-x_{1}\right)} d x_{1} \\
& =(1 / 2)^{\beta}+\beta \int_{1 / 2}^{1}\left(x^{-\alpha \beta}\left(1+(2 x-1)^{\alpha \beta}\right)\right)^{-1 / \alpha-1} x^{-\alpha \beta-1} d x .
\end{aligned}
$$

Now we choose $\alpha=1 / \beta$ (for this choice of $\alpha$ and $\beta$ one can explicitly calculate $\left.q_{2,1}^{F}\right)$. Hence

$$
q_{2,1}^{F}(1 / \beta, \beta)=(1 / 2)^{\beta}+\beta \int_{1 / 2}^{1} 2^{-\beta-1} x^{-2} d x=(1 / 2)^{\beta}[1+\beta / 2] .
$$

Using (5.4), this implies that

$$
q_{2,1}^{F}(1 / \beta, \beta)=(1 / 2)^{\beta}[1+\beta] .
$$

In motor liability, often $\beta=2$ is an appropriate choice for extreme value considerations. So if we have two motor portfolios with decay parameter $\beta=2$ and dependence parameter $\alpha=1 / 2$ (which corresponds to a tail dependence

\section{Fréchet case}

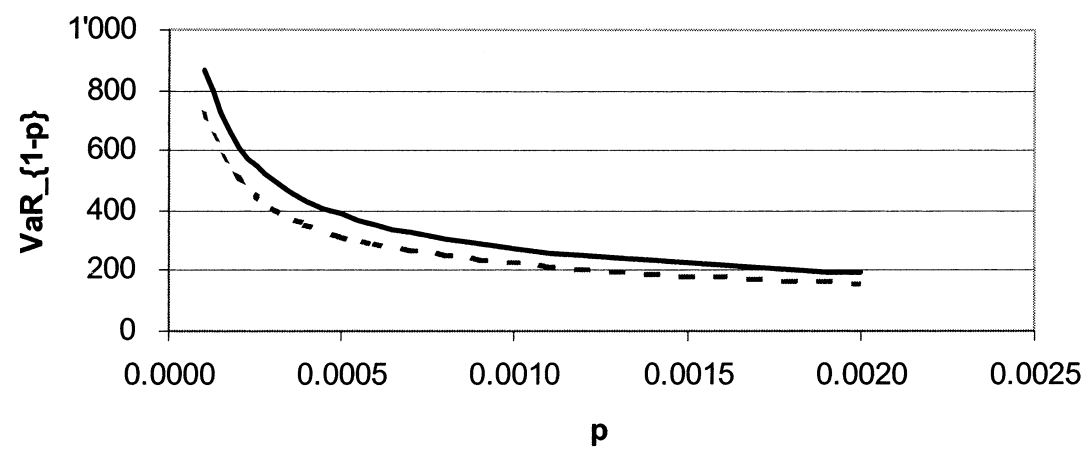

- - - - Independent ——Regularly Varying

Figure 1: Fréchet case: asymptotic behaviour of $\operatorname{VaR}_{1-p}\left(Y_{1}+Y_{2}\right)$ and $\operatorname{VaR}_{1-p}\left(X_{1}+X_{2}\right)$ as a function of $p(\theta=5)$. 
coefficient $\lambda=1 / 4$ (see Theorem 3.9 in [8])) we obtain $q_{2}^{F}(1 / 2,2)=3 / 4$. Hence for such a portfolio we obtain

$$
P\left[X_{1}+X_{2} \leq-2 u\right] \sim \frac{3}{4} \cdot\left(\frac{\theta}{u}\right)^{2} \text { as } u \rightarrow \infty .
$$

This means that the asymptotic behaviour of the Value-at-Risk is ( $p$ small)

$$
\operatorname{VaR}_{1-p}\left(X_{1}+X_{2}\right) \sim \theta(3 / p)^{1 / 2} \text { as } p \rightarrow 0 \text {. }
$$

We compare this to the independent case: Assume $-Y_{1}$ and $-Y_{2}$ are i.i.d. Pareto distributed with parameter $\beta \geq 1$. Hence

$$
\begin{aligned}
P\left[Y_{1}+Y_{2} \leq-2 u\right] & =P\left[Y_{1} \leq-2 u+\theta\right]+P\left[Y_{1} \geq-2 u+\theta, Y_{2} \leq-2 u-Y_{1}\right] \\
& =\left(\frac{2 u-\theta}{\theta}\right)^{-\beta}+\int_{-2 u+\theta}^{-\theta} \frac{\beta}{\theta}\left(\frac{-x}{\theta}\right)^{-\beta-1}\left(\frac{2 u+x}{\theta}\right)^{-\beta} d x \\
& \sim 2\left(\frac{\theta}{2 u}\right)^{\beta} \text { as } u \rightarrow \infty .
\end{aligned}
$$

Hence for $\beta=2$ we have

$$
\operatorname{VaR}_{1-p}\left(Y_{1}+Y_{2}\right) \sim \theta(2 / p)^{1 / 2}
$$

which is strictly smaller than $\operatorname{VaR}_{1-p}\left(X_{1}+X_{2}\right)$ (see Figure 1).

\section{Example 5.2 (Gumbel case)}

We choose standard Gaussian marginals and archimedean copula generated by $\psi \in \Re_{-\alpha}^{0+}$ with $\alpha>0$. In view of (4.5)

$$
\begin{aligned}
q_{2,1}^{G}(\alpha) & =P\left[\sum_{i=1}^{2} \tilde{Z}_{i}^{(1)} \leq 2\right]=\left.\int_{-\infty}^{1} \frac{d G_{2,1}^{(\alpha)}(x, y)}{d x}\right|_{(x, y)=\left(x_{1}, 2-x_{1}\right)} d x_{1} \\
& =\int_{-\infty}^{0}(2 \cosh (\alpha x))^{-1 / \alpha-1} \exp \{-\alpha x\} d x .
\end{aligned}
$$

This can be determined numerically, e.g. $q_{2,1}^{G}(1)=0.6427$. This implies, using (5.4),

$$
q_{2}^{G}(1)=2 \cdot q_{2,1}^{G}(1)-1 / 2=0.7854 \text {. }
$$

Using Theorem 3.3 and because of (5.2), we obtain for the asymptotic behaviour $(\alpha=1)$

$$
P\left[X_{1}+X_{2} \leq-2 u\right] \sim \frac{q_{2}^{G}(1)}{(2 \pi)^{1 / 2}} \cdot \frac{1}{u} \cdot e^{-u^{2} / 2} \text { as } u \rightarrow \infty .
$$


We compare this to the bivariate Gaussian case: Assume $\left(Y_{1}, Y_{2}\right)$ is bivariate normally distributed with standard Gaussian marginals and correlation $\rho \in(0,1)$. Hence $Y_{1}+Y_{2}$ is Gaussian distributed with mean 0 and variance $\sigma^{2}=2(1+\rho)$. Because of (5.2) this implies that

$$
\begin{aligned}
P\left[Y_{1}+Y_{2} \leq-2 u\right] & =P\left[\frac{Y_{1}+Y_{2}}{\sigma} \leq \frac{-2 u}{\sigma}\right]=\Phi\left(-\frac{2^{1 / 2} u}{(1+\rho)^{1 / 2}}\right) \\
& \sim\left(\frac{1+\rho}{4 \pi}\right)^{1 / 2} \cdot \frac{1}{u} \cdot e^{-u^{2} /(1+\rho)} \quad \text { as } u \rightarrow \infty .
\end{aligned}
$$

Hence we have a faster decay in the bivariate normal case than in the regulary varying archimedean case. Of course this is not surprising, since we know from [9], Theorem 5.3, that the bivariate gaussian distribution is asymptotically independent in a distributional sense for all $\rho \in[0,1$ ) (see also [4], Section 4.4). For $\rho=1 / 2$ and $\alpha=1$ we obtain for the asymptotic Value-at-Risk behaviour the picture given in Figure 2.

\section{Gumbel case}

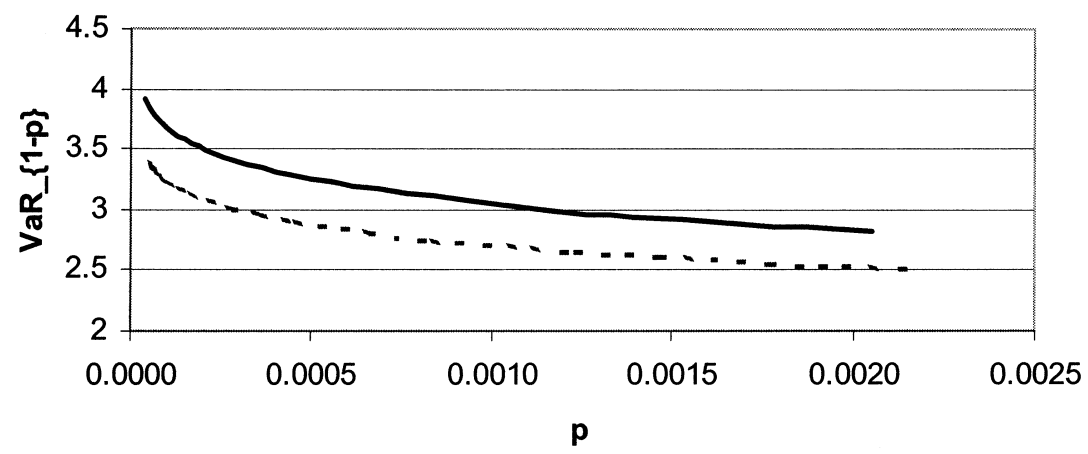

- - - Bivariate Gaussian —_Regularly Varying

Figure 2: Gumbel case: asymptotic behaviour of $\operatorname{VaR}_{1-p}\left(Y_{1}+Y_{2}\right)$ and $\operatorname{VaR}_{1-p}\left(X_{1}+X_{2}\right)$ as a function of $p(\rho=1 / 2)$.

\subsection{Conclusions}

In many applied situations we are confronted with the question of a suitable Value-at-Risk estimate for dependent random variables. Usually, e.g. in actuarial problems, one faces the additional problem that one has not enough data to really analyze the dependence structure in the tails. For such situations Theorem 3.3 and Lemmas 6.1, 7.1, 7.2 are appropriate tools for tail estimates. All that we have to specify is the dependence strength $\alpha$ and the marginal 
behaviour $F$. From this information we are already able to estimate the asymptotic Value-at-Risk (for estimating $\alpha$ we refer to [4] and [8]).

In the two examples, we have seen that the dependence structure has a rather large impact on joint extreme value calculations (it blows up the Valueat-Risk of aggregate dependent random variables by a factor larger than 1 compared to the independent situation). In practice one often chooses an independent or a Gaussian model (maybe after logarithmizing the data). Both models are asymptotically independent (see [9]), and hence one has to be very careful with the application of such models to practical situations, because they tend to underestimate joint extremes. Our examples have indicated what the difference between a mulitivariate dependent and an independent model can be. The question which remains open is: How fast is the convergence? Of course, for answering this question we would have to make more assumptions on the dependence structure. In particular, in our archimedean situation one would have to analyze higher order terms of the regularly varying generators $\psi$.

\section{Proofs in the Fréchet Case}

We want to point out that the crucial result is formula (6.2). It shows that due to the dependence structure, one coordinate already determines the asymptotic behaviour of the whole random vector. For the random vector $\left(X_{1}, \ldots, X_{d}\right)$, $k \leq d$, we define the event

$$
A_{k}(u)=\left\{X_{1} \leq-u, \ldots, X_{k} \leq-u\right\}
$$

Lemma 6.1 (Fréchet case) Assume $d \geq 2, \alpha>0$ and $\beta>0$. Choose $\boldsymbol{X}$ satisfying Assumption 3.1 with $\psi \in \mathfrak{R}_{-\alpha}^{0+}$ and $F(\cdot) \in \mathfrak{R}_{-\beta}^{-\infty}$. For $1 \leq k \leq d, x_{1}, \ldots, x_{k} \in[0,1]$ and $x_{k+1}, \ldots, x_{d} \geq 0$ the following limits are well-defined and we have

$$
\begin{aligned}
& \lim _{u \rightarrow \infty} P\left[X_{1} \leq-u / x_{1}, \ldots, X_{d} \leq-u / x_{d} \mid A_{k}(u)\right]=H_{d, k}^{(\alpha, \beta)}\left(x_{1}, \ldots, x_{d}\right), \\
& \lim _{u \rightarrow \infty} P\left[\sum_{i=1}^{d} X_{i} \leq-u d \mid A_{k}(u)\right]=q_{d, k}^{F}(\alpha, \beta) .
\end{aligned}
$$

Proof of Lemma 6.1. First we show that the argument on the left-hand side of (6.2)-(6.3) is well-defined. Choose $u>0$. For $\varepsilon>0$ there exists $u_{0} \geq u$ such that

$$
\begin{aligned}
P\left[A_{k}(u)\right] & \geq P\left[A_{k}\left(u_{0}\right)\right]=P\left[X_{1} \leq-u_{0}, \ldots, X_{k} \leq-u_{0}\right] \\
& =\psi^{-1}\left(k \psi \circ F\left(-u_{0}\right)\right) \geq \psi^{-1} \circ \psi\left((k+\varepsilon)^{-1 / \alpha} F\left(-u_{0}\right)\right) \\
& =(k+\varepsilon)^{-1 / \alpha} F\left(-u_{0}\right)>0,
\end{aligned}
$$

where we have used Sklar's theorem and $\psi \in \mathfrak{R}_{-\alpha}^{0+}$. Hence the argument on the left-handside of (6.2)-(6.3) is well-defined. For $1 \leq k \leq d$, we choose $x_{1}, \ldots, x_{k} \leq 1$. We define for $u>0$ 


$$
\begin{aligned}
F_{u}^{(k)}\left(x_{1}, \ldots, x_{d}\right) & =P\left[X_{1} \leq-u / x_{1}, \ldots, X_{d} \leq-u / x_{d} \mid A_{k}(u)\right] \\
& =P\left[\frac{-u}{X_{1}} \leq x_{1}, \ldots, \frac{-u}{X_{d}} \leq x_{d} \mid \frac{-u}{X_{1}} \leq 1, \ldots, \frac{-u}{X_{k}} \leq 1\right] .
\end{aligned}
$$

$F_{u}^{(k)}$ defines a distribution on $[0,1]^{k} \times[0, \infty)^{d-k}$. We use the following abbreviation $\mathbf{Y}^{(u)}=\left(Y_{1}^{(u)}, \ldots, Y_{d}^{(u)}\right) \stackrel{d}{\sim} F_{u}^{(k)}$ i.e. $\mathbf{Y}^{(u)}$ has the same distribution as $\left(-u / X_{1}, \ldots,-u / X_{d}\right)$ conditioned on the event $A_{k}(u)$. We prove a weak convergence result for $\mathbf{Y}^{(u)}$.

Since $F(\cdot) \in \Re_{-\beta}^{-\infty}$ we have for $x_{i}$ as above and $\varepsilon>0$ that there exists $u_{0}$ such that for all $u>u_{0}$

$$
\left(x_{i}-\varepsilon\right)^{\beta} F(-u) \leq F\left(-u / x_{i}\right) \leq\left(x_{i}+\varepsilon\right)^{\beta} F(-u) .
$$

Since $\psi$ is regularly varying with index $-\alpha$, we get a similar estimate for $\psi$ (see (6.4)). This implies for all $\varepsilon>0$, using Sklar's Theorem 2.2,

$$
\begin{aligned}
& \limsup _{u \rightarrow \infty} F_{u}^{(k)}\left(x_{1}, \ldots, x_{d}\right)=\limsup _{u \rightarrow \infty} \frac{\psi^{-1}\left(\sum_{i=1}^{d} \psi \circ F\left(-u / x_{i}\right)\right)}{\psi^{-1}(k \psi \circ F(-u))} \\
& \leq \limsup _{u \rightarrow \infty} \frac{\psi^{-1}\left(\sum_{i=1}^{d} \psi\left(\left(x_{i}+\varepsilon\right)^{\beta} F(-u)\right)\right)}{\psi^{-1}(k \psi \circ F(-u))} \leq \limsup _{u \rightarrow \infty} \frac{\psi^{-1}\left(\sum_{i=1}^{d}\left(x_{i}+2 \varepsilon\right)^{-\alpha \beta} \psi(F(-u))\right)}{(k+\varepsilon)^{-1 / \alpha} F(-u)} \\
& \leq \limsup _{u \rightarrow \infty} \frac{\psi^{-1}\left(\psi\left(\left(\sum_{i=1}^{d}\left(x_{i}+2 \varepsilon\right)^{-\alpha \beta}-\varepsilon\right)^{-1 / \alpha} F(-u)\right)\right)}{(k+\varepsilon)^{-1 / \alpha} F(-u)} \\
& =\left(\frac{\sum_{i=1}^{d}\left(x_{i}+2 \varepsilon\right)^{-\alpha \beta}-\varepsilon}{k+\varepsilon}\right) .
\end{aligned}
$$

Using the same arguments we have a similar lower bound for the lim inf. Since $\varepsilon$ was chosen arbitrarily, this proves (6.2):

$$
\begin{aligned}
& \lim _{u \rightarrow \infty} F_{u}^{(k)}\left(x_{1}, \ldots, x_{d}\right)=\lim _{u \rightarrow \infty} P\left[X_{1} \leq-u / x_{1}, \ldots, X_{d} \leq-u / x_{d} \mid A_{k}(u)\right] \\
& =H_{d, k}^{(\alpha, \beta)}\left(x_{1}, \ldots, x_{d}\right) .
\end{aligned}
$$

$H_{d, k}^{(\alpha, \beta)}$ is a continuous function. Because $F_{u}^{(k)}$ is a distribution on $[0,1]^{k} \times[0, \infty)^{d-k}$ so is $H_{d, k}^{(\alpha, \beta)}$. This means that $\mathbf{Y}^{(u)}$ converges weakly to $\mathbf{Y}=\left(Y_{1}, \ldots, Y_{d}\right) \stackrel{d}{\sim} H_{d, k}^{(\alpha, \beta)}$ as $u \rightarrow \infty$. In view of the continuous mapping theorem (see e.g. Theorem A2.6 in [3]) this implies

$$
\begin{aligned}
& \lim _{u \rightarrow \infty} P\left[\sum_{i=1}^{d} X_{i} \leq-u d \mid A_{k}(u)\right]=\lim _{u \rightarrow \infty} P\left[\sum_{i=1}^{d} \frac{1}{Y_{i}^{(u)}} \geq d\right] \\
& =P\left[\sum_{i=1}^{d} \frac{1}{Y_{i}} \geq d\right]=q_{d, k}^{F}(\alpha, \beta) .
\end{aligned}
$$

This proves Lemma 6.1 . 
Proof of Theorem 3.3 in the Fréchet case: Because of Assumption 3.1 we have exchangeability. Hence the following identity is true:

$$
P\left[\bigcup_{k=1}^{d}\left\{X_{k} \leq-u\right\}\right]=\sum_{k=1}^{d}(-1)^{k-1}\left(\begin{array}{l}
d \\
k
\end{array}\right) P\left[A_{k}(u)\right]
$$

Hence using exchangeability once again we obtain

$$
\begin{aligned}
P\left[\sum_{i=1}^{d} X_{i} \leq-u d\right] & =P\left[\sum_{i=1}^{d} X_{i} \leq-u d, \bigcup_{k=1}^{d}\left\{X_{k} \leq-u\right\}\right] \\
& =\sum_{k=1}^{d}(-1)^{k-1}\left(\begin{array}{l}
d \\
k
\end{array}\right) P\left[\sum_{i=1}^{d} X_{i} \leq-u d, A_{k}(u)\right] \\
& =\sum_{k=1}^{d}(-1)^{k-1}\left(\begin{array}{l}
d \\
k
\end{array}\right) P\left[\sum_{i=1}^{d} X_{i} \leq-u d \mid A_{k}(u)\right] P\left[A_{k}(u)\right] .
\end{aligned}
$$

Dividing (6.11) by $F(-u)$ we have that (3.3) follows from (6.9) and (6.4). There remains to prove that $q_{d}^{F}(\alpha, \beta)$ is positive:

$$
\liminf _{u \rightarrow \infty} \frac{1}{F(-u)} P\left[\sum_{i=1}^{d} X_{i} \leq-u d\right] \geq \liminf _{u \rightarrow \infty} \frac{P\left[A_{d}(u)\right]}{F(-u)}=d^{-1 / \alpha}>0 .
$$

\section{Proofs in the Weibull and Gumbel Case}

For the random vector $\left(X_{1}, \ldots, X_{d}\right), k \leq d$, and $x_{F}$ we define the event

$$
\tilde{A}_{k}(u)=\left\{X_{1} \leq x_{F}+1 / u, \ldots, X_{k} \leq x_{F}+1 / u\right\} .
$$

Lemma 7.1 (Weibull case) Assume $d \geq 2, \alpha>0$ and $\beta>0$. Choose $\mathbf{X}$ satisfying Assumption 3.1 with $\psi \in \mathfrak{R}_{-\alpha}^{0+}, x_{F}>-\infty$ and $F\left(x_{F}-1 / \cdot\right) \in \mathfrak{R}_{-\beta}^{-\infty}$. For $1 \leq k \leq d, x_{1}$, $\ldots, x_{k} \in[0,1]$ and $x_{k+1}, \ldots, x_{d} \geq 0$ the following limits are well-defined and we have

$$
\begin{aligned}
& \lim _{u \rightarrow \infty} P\left[X_{1} \leq x_{F}+x_{1} / u, \ldots, X_{d} \leq x_{F}+x_{d} / u \mid \tilde{A}_{k}(u)\right]=H_{d, k}^{(\alpha, \beta)}\left(x_{1}, \ldots, x_{d}\right),(7,2) \\
& \lim _{u \rightarrow \infty} P\left[\sum_{i=1}^{d} X_{i} \leq d\left(x_{F}+1 / u\right) \mid \tilde{A}_{k}(u)\right]=q_{d, k}^{W}(\alpha, \beta)
\end{aligned}
$$

Proof of Lemma 7.1: As in (6.4) we have $\mathrm{P}\left[\tilde{A}_{k}(u)\right]>0$, hence the arguments in (7.2)-(7.3) are well-defined. Next we define for $u>0$

$$
\begin{aligned}
F_{u}^{(k)}\left(x_{1}, \ldots, x_{d}\right) & =P\left[X_{1} \leq x_{F}+x_{1} / u, \ldots, X_{d} \leq x_{F}+x_{d} / u \mid \tilde{A}_{k}(u)\right] \\
& =P\left[u\left(X_{1}-x_{F}\right) \leq x_{1}, \ldots, u\left(X_{d}-x_{F}\right) \leq x_{d} \mid \tilde{A}_{k}(u)\right] .
\end{aligned}
$$


$F_{u}^{(k)}$ is a distribution with the first $k$ coordinates bounded by 1 . For $x_{i}>0, \varepsilon>0$ there exists $u_{0}$ such that for all $u>u_{0}$

$$
\left(x_{i}-\varepsilon\right)^{\beta} F\left(x_{F}+1 / u\right) \leq F\left(x_{F}+x_{i} / u\right) \leq\left(x_{i}+\varepsilon\right)^{\beta} F\left(x_{F}+1 / u\right) .
$$

This implies as in (6.7)-(6.8)

$$
\begin{aligned}
\lim _{u \rightarrow \infty} F_{u}^{(k)}\left(x_{1}, \ldots, x_{d}\right) & =\lim _{u \rightarrow \infty} \frac{\psi^{-1}\left(\sum_{i=1}^{d} \psi \circ F\left(x_{F}+x_{i} / u\right)\right)}{\psi^{-1}\left(k \psi \circ F\left(x_{F}+1 / u\right)\right)} \\
& =H_{d, k}^{(\alpha, \beta)}\left(x_{1}, \ldots, x_{d}\right) .
\end{aligned}
$$

Therefore we observe weak convergence which together with the continuous mapping theorem implies

$$
\begin{aligned}
\lim _{u \rightarrow \infty} P & {\left[\sum_{i=1}^{d} X_{i} \leq d\left(x_{F}+1 / u\right) \mid \tilde{A}_{k}(u)\right] } \\
& =\lim _{u \rightarrow \infty} P\left[\sum_{i=1}^{d} u\left(X_{i}-x_{F}\right) \leq d \mid \tilde{A}_{k}(u)\right]=q_{d}^{W}(\alpha, \beta) .
\end{aligned}
$$

This finishes the proof of Lemma 7.1.

For the random vector $\left(X_{1}, \ldots, X_{d}\right), k \leq d$, and a function a $(\cdot)$ we define the event

$$
\hat{A}_{k}(u)=\left\{X_{1} \leq u+a(u), \ldots, X_{k} \leq u+a(u)\right\} .
$$

Lemma 7.2 (Gumbel case) Assume $d \geq 2$ and $\alpha>0$. Choose $\mathbf{X}$ satisfying Assumption 3.1 with $\psi \in \Re_{-\alpha}^{0+}, x_{F} \geq-\infty$ and there exists a positive function a $(\cdot)$ such that (3.2) is satisfied. For $1 \leq k \leq d, x_{1}, \ldots, x_{k} \in[0,1]$ and $x_{k+1}, \ldots, x_{d} \geq 0$ the following limits are well-defined and we have

$$
\begin{aligned}
& \lim _{u \rightarrow \infty} P\left[X_{1} \leq u+x_{1} a(u), \ldots, X_{d} \leq u+x_{d} a(u) \mid \tilde{A}_{k}(u)\right]=G_{d, k}^{(\alpha)}\left(x_{1}, \ldots, x_{d}\right), \\
& \lim _{u \rightarrow \infty} P\left[\sum_{i=1}^{d} X_{i} \leq d(u+a(u)) \mid \tilde{A}_{k}(u)\right]=q_{d, k}^{G}(\alpha) .
\end{aligned}
$$

Proof of Lemma 7.2: As in (6.4) we have $P\left[\hat{A}_{k}(u)\right]>0$, hence the arguments in (7.9)-(7.10) are well-defined. Next we define

$$
\begin{aligned}
F_{u}^{(k)}\left(x_{1}, \ldots, x_{d}\right) & =P\left[X_{1} \leq u+x_{1} a(u), \ldots, X_{d} \leq u+x_{d} a(u) \mid \hat{A}_{k}(u)\right] \\
& =P\left[\frac{X_{1}-u}{a(u)} \leq x_{1}, \ldots, \frac{X_{d}-u}{a(u)} \leq x_{d} \mid \hat{A}_{k}(u)\right] .
\end{aligned}
$$


$F_{u}^{(k)}$ is a distribution with the first $k$ coordinates bounded by 1 . For $x_{i} \in \mathbb{R}, \varepsilon>0$ there exists $u_{0}>x_{F}$ such that for all $u \in\left(x_{F}, u_{0}\right)$

$$
e^{x_{i}-\varepsilon} F(u) \leq F\left(u+x_{i} a(u)\right) \leq e^{x_{i}+\varepsilon} F(u) .
$$

This implies as in (6.7)-(6.8)

$$
\begin{aligned}
& \lim _{u \rightarrow \infty} F_{u}^{(k)}\left(x_{1}, \ldots, x_{d}\right)=\lim _{u \rightarrow \infty} \frac{\psi^{-1}\left(\sum_{i=1}^{d} \psi \circ F\left(u+x_{i} a(u)\right)\right)}{\psi^{-1}(k \psi \circ F(u+a(u)))} \\
& =\lim _{u \rightarrow \infty} \frac{\psi^{-1} \circ \psi\left(\left(\sum_{i=1}^{d} \exp \left\{-\alpha\left(x_{i}-1\right)\right\}\right)^{-1 / \alpha} F(u+a(u))\right)}{k^{-1 / \alpha} F(u+a(u))}=G_{d, k}^{(\alpha)}\left(x_{1}, \ldots, x_{d}\right) .
\end{aligned}
$$

But then the proof follows as in the Fréchet and Weibull case. This finishes the proof of Lemma 7.2.

Proof of Theorem 3.3 in the Weibull and Gumbel case (3.4)-(3.5): The proofs are similar to (6.10)-(6.11): replace $\left\{X_{k} \leq-\mathrm{u}\right\}$ by $\left\{X_{k} \leq x_{F}+1 / u\right\}$ and $\left\{X_{k} \leq u+a(u)\right\}$, resp., and replace the event $A_{k}(u)$ by $\tilde{A}_{k}(u)$ and $\hat{A}_{k}(u)$, resp. This finishes the proof of Theorem 3.3.

Proof of Lemma 4.1. Formulas (6.8) and (7.13) imply that $H_{d, k}^{(\alpha, \beta)}$ and $G_{d, k}^{(\alpha)}$ are continuous distributions. Hence there remains to show that they have Clayton copula. We only prove the statement for $H_{d, k}^{(\alpha, \beta)}$ since for $G_{d, k}^{(\alpha)}$ the proof is analogous.

First we determine the marginals: $1 \leq j \leq k \leq i \leq d$

$$
\begin{aligned}
& H_{j}\left(x_{j}\right)=\left(\frac{k-1+x_{j}^{-\alpha \beta}}{k}\right)^{-1 / \alpha}, H_{j}^{-1}\left(y_{j}\right)=\left(k y_{j}^{-\alpha}-(k-1)\right)^{-1 / \alpha \beta}, \\
& H_{i}\left(x_{i}\right)=\left(\frac{k+x_{i}^{-\alpha \beta}}{k}\right)^{-1 / \alpha}, H_{i}^{-1}\left(y_{i}\right)=\left(k y_{i}^{-\alpha}-k\right)^{-1 / \alpha \beta} .
\end{aligned}
$$

Sklar's Theorem 2.2 immediately implies

$$
H_{d, k}^{(\alpha, \beta)}\left(H_{1}^{-1}\left(x_{1}\right), \ldots, H_{d}^{-1}\left(x_{d}\right)\right)=C^{\mathrm{Cl}, \alpha}\left(x_{1}, \ldots, x_{d}\right) .
$$

This proves Lemma 4.1.

\section{REFERENCES}

[1] BäUerle, N. and Müller, A. (1998) Modelling and comparing dependencies in multivariate risk portfolios. Astin Bulletin 28, 1, 59-76.

[2] Bingham, N.H., Goldie, C.M. and Teugels, J.L. (1987) Regular variation. Cambridge University Press, Cambridge. 
[3] Embrechts, P., Klüppelberg, C. and Mikosch, T. (1997) Modelling extremal events for insurance and finance. Springer, Berlin.

[4] Embrechts, P., McNeil, A. and Straumann, D. (2002) Correlation and Dependency in Risk Management: Properties and Pitfalls. In Risk Management: Value at Risk and Beyond, M. Dempster (Ed.), Cambridge University Press, Cambridge, 176-223.

[5] Frees, W.E. and VAldeZ, E.A. (1998) Understanding Relationships Using Copulas. North American Actuarial Journal 2, 1-25.

[6] Hürlimann, W. (2002) Fitting bivariate cumulative returns with copulas. Proceedings of the 33rd International Astin Colloquium, Cancun, Mexico.

[7] JoE, H. (1997) Multivariate Models and Dependence Concepts. Chapman \& Hall, London.

[8] JURI, A. and WÜTHRICH, M.V. (2002) Copula convergence theorems for tail events. Insurance: Math. Econom. 30, 405-420.

[9] JuRI, A. and WÜthrich, M.V. (2002) Tail dependence from a distributional point of view. Preprint.

[10] Kimberling, C. (1974) A probabilistic interpretation of complete monotonicity. Aequationes Mathematicae 10, 152-164.

[11] Klugman, S.A. and Prasa, R. (1999) Fitting bivariate loss distributions with copulas. Insurance: Math. Econom. 24, 139-148.

[12] KREMER, E. (1998) Largest claims reinsurance premiums under possible claims dependence. Astin Bulletin 28/2, 257-267.

[13] Frey, R. and McNeil A.J. (2001) Modelling dependent defaults, Preprint.

[14] Nelsen, R.B. (1999) An introduction to copulas. Springer, New York.

[15] Schweizer, B. and Sklar, A. (1983) Probabilistic Metric Spaces, North-Holland, New York.

[16] Sklar, A. (1959) Fonctions de répartition aux dimensions et leurs marges. Publications de l'Institut de Statistique de l'Université de Paris 8, 229-231.

\author{
MARIO V. WÜTHRICH \\ Winterthur Insurance \\ Römerstrasse 17 \\ P.O. Box 357 \\ CH-8401 Winterthur \\ Switzerland \\ mario.wuethrich@winterthur.ch
}

